\title{
Editorial for the Special Issue: Green Cognitive and Cooperative Communication and Networking
}

\author{
Lin Chen • Wei Wang • Alagan S. Anpalagan • \\ Athanasios V. Vasilakos
}

Published online: 15 March 2013

(C) Springer Science+Business Media New York 2013

Wireless communication has nowadays become one of the major worldwide causes of energy consumption in the field of ICT, with a devastating impact in terms of pollution and energy waste. As a result, the past decade has witnessed tremendous efforts and progress made by both the industry and academia for improving energy and power efficiency in current and emerging wireless communication networks, among which cognitive and cooperative communication are proposed as key technologies to increase both spectrum and energy efficiency. With cognitive and cooperative communication, the use of larger spectrum band and the opportunistic adaptation of the spectrum use lead to more effective interference management, better spatial and temporal reuse, thus reducing the power consumption. Despite the ever growing interests, the research on green cognitive and cooperative communication and networking is still in its infancy. Some fundamental problems are still open and require immediate studies.

This special issue is intended to provide a forum for presenting, exchanging and discussing the recent advances on green cognitive and cooperative communication and networking. In response to the call for paper, we have received 26 paper submissions from both academia and industries that covered a variety of topics. Two rounds of

\section{Chen $(\triangle)$}

Department of Computer Science, University of Paris-Sud, Orsay, France

e-mail: Lin.Chen@1ri.fr

W. Wang

Department of Information Science and Electronic Engineering, Zhejiang University, Hangzhou, China

\section{A. S. Anpalagan}

Department of Electrical and Computer Engineering,

Ryerson University, Toronto, ON, Canada

A. V. Vasilakos

University of Western Macedonia, Kozani, Greece careful review by the guest editors and experts in the field led to 5 papers for inclusion in this issue.

In the first article, "Green Cooperative Cognitive Communication and Networking: A New Paradigm for Wireless Networks", the authors (Lin Chen, Wei Wang, Alagan S. Anpalagan, Athanasios V. Vasilakos, Kandasamy Illanko, Honggang Wang, Muhammad Naeem) provide a comprehensive survey of the green cognitive and cooperative communication and networking techniques from its characteristics point of view to operational details in the eventual deployment. They present a systematic overview on the tools and techniques that can be used to solve problems arising in energy efficiency optimization problem in this context. The need to incorporate green concepts such as multi-input and multi-output, multirate, and multi-carrier systems, short-range low-power communication using small cell networks, and machine to machine communication in emerging and advanced wireless communication technologies is also addressed. Finally, the paper highlights design challenges and open issues in embracing green technologies in different and cross layers of communication and networking.

In the second paper titled "Towards Energy-Efficiency in Selfish, Cooperative Networks", the authors (Chi Harold Liu, Jun Fan, Zhengguo Sheng, Xiumei Fan, Kin K. Leung) propose an adaptive multi-relay selection with power allocation mechanism to offer energy fairness at each node for a cooperative network. Unlike traditional approaches where all nodes are considered to transmit in a collaborative manner, they explicitly consider the situation where nodes exhibit some degree of selfish behavior. Specifically, they introduce a novel concept of the selfishness index and incorporate it into a utility function which denotes the degree a node can benefit from cooperative transmission. Theoretical analysis and simulation results are supplemented to show advantages in maximizing the network lifetime and guaranteeing the QoS in realistic wireless environments. They also consider the practical situation when nodes consume energy in mode switching, and study the 
behavior of inter-cluster relay switching and the trade-off among network lifetime, switching cost and switching frequency.

In the third paper, "Green Access Point Selection for Wireless Local Area Networks Enhanced by Cognitive Radio", the authors (Xi Li, Wendong Ge, Shanzhi Chen, Hong Ji, Victor C.M. Leung) propose an optimal Green AP Selection (GAPS) scheme, in which AP selection is optimized to maximize the system throughput while minimizing the energy consumption, for multi-rate WLANs enhanced by CRs. Different from most existing AP selection schemes, GAPS takes into account of the state transition tendency of APs and the influence of Automatic Rate Fallback mechanism in the Distributed Coordination Function. The AP selection problem is formulated as a restless bandit problem and solved by the primal-dual index heuristic algorithm based on first order relaxation to yield the GAPS scheme with the "indexability" property and hence a low complexity. GAPS is further divided into offline computation, which accounts for the bulk of the computations, and online selection, with a low complexity to facilitate implementation. Simulation results illustrate the performance improvements of GAPS compared with existing AP selection schemes in different scenarios.

In the fourth paper, "Energy-Efficiency Study for Twotier Heterogeneous Networks (HetNet) under Coverage Performance Constraints", the authors (Xing Zhang, Zhuowen $\mathrm{Su}$, Zhi Yan, Wenbo Wang) study the optimal energy efficiency of a two-tier heterogeneous network that consists of a macro-cell and many small cells under coverage performance constraints for different spectrum deployments. They first derive the closed form expressions of coverage performance for each tier based on stochastic geometry. Then the relationship between energy efficiency and the density of small cells for the two-tier network are evaluated, the optimal density of small cells that maximize energy efficiency under coverage performance constraints for the two-tier network is obtained.

In the last paper, "Interference Management and Power Allocation for Energy-Efficient Cognitive Femtocell Networks", the authors (F. Richard Yu, Renchao Xie, Hong Ji) study the energy efficiency aspect of power allocation and interference management in heterogeneous cognitive radio networks with femtocells. Particularly, due to sharing the same spectrum resource for cross-tier cognitive radio networks with femtocells, there is cross-tier interference between fetmocells and macrocell. In this case, they introduce an interference price to measure the effect of interference. Then they formulate the problem of power allocation and interference management for energy-efficient transmissions in heterogeneous cognitive radio networks with femtocells as a Stackelberg game. The backward induction method is used to solve the optimal power allocation and price determination. An iteration algorithm based on price updating is proposed to obtain the Stackelberg equilibrium solution. Simulation results are illustrated to demonstrate the Stackelberg equilibrium by the proposed iteration algorithm, and energy efficiency can be improved in the proposed scheme.

We would like to thank all the reviewers for their efforts and constructive comments. We would also like to thank Professor Imrich Chlamtac, the Editor-in-Chief, for his support and Dr. Sara Fruner and Ms. Ronila Gamatero for their efforts during the entire processing stages of the special issue. Finally, we would like to thank all the authors who submitted their precious research work to this special issue.

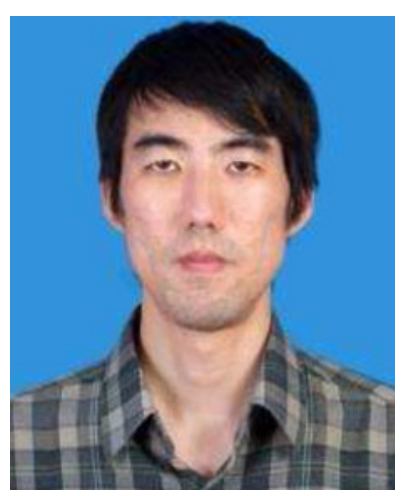

Dr. Lin Chen received his B.E. degree in Radio Engineering from Southeast University, China in 2002, the Engineer and Ph.D. degrees from Telecom ParisTech, Paris in 2005 and 2008. He also holds a M.S. degree of Networking from the University of Paris 6. He currently works as associate professor in the Department of Computer Science of the University of Paris-Sud. His main research interests include cognitive radio networks, cooperative communication, game-theoretic modeling and protocol design for wireless networks, network security and cooperation enforcement. He has published more than 20 journal/conference articles and book chapters. He has served as TPC member of several major conferences on networking and communications.

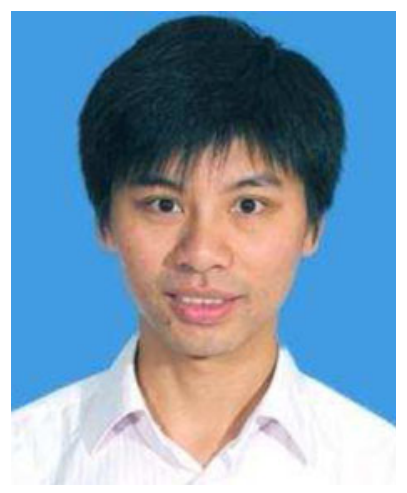

Dr. Wei Wang received his B.S. degree in Communication Engineering and Ph.D. degree in Signal and Information Processing in 2004 and 2009, respectively, both from Beijing University of Posts and Telecommunications, China. Now, he is an associate professor with the Department of Information Science and Electronic Engineering, Zhejiang University, China. From 2007 to 2008 , he visited the RealTime Computing Lab of the University of Michigan, Ann Arbor, USA. Since 2013, he began to visit Hong Kong University of Science and Technology, Hong Kong. His research interest mainly focuses on cognitive radio networks, spectrum aggregation, green communications, cooperative communications, and radio resource management for wireless networks. He is the editor of the book "Cognitive Radio Systems" (Intech, 2009) and serves as an editor for Transactions on 
Emerging Telecommunications Technologies. He serves as a TPC co-chair for CRNet 2010 and NRN 2011, Symposium co-chair for WCSP 2013, and also serves as a TPC member for major international conferences.

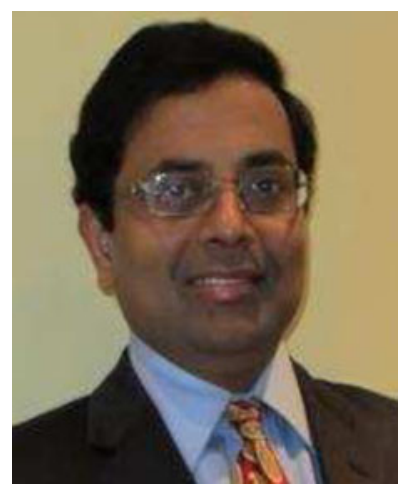

Dr. Alagan S. Anpalagan received B.A.Sc., M.A.Sc. and Ph.D. degrees in Electrical Engineering from the University of Toronto, Canada. He is currently Full Professor in the Department of Electrical and Computer Engineering at Ryerson University. His current research interests include cognitive radio resource allocation and management, wireless cross layer design and optimization, collaborative communication, green communications technologies, machine-to-machine communication, and small cell networks. Dr. Anpalagan has published more than 150 technical papers. He serves as Associate Editor for the IEEE Communications Surveys \& Tutorials (2012-), IEEE Communications Letters (2010-) and Springer Wireless Personal Communications (2009-), and past Editor for EURASIP Journal of Wireless Communications and Networking
(2004-2009). He served as the general chair, TPC chair and symposium chair for many international conferences.

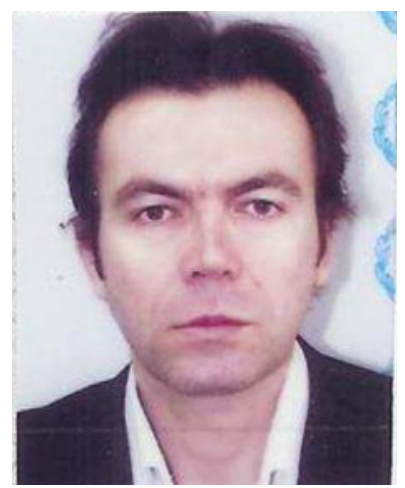

Dr. Athanasios V. Vasilakos is currently Professor at the University of Western Macedonia, Greece. He has authored or coauthored over 200 technical papers in major international journals and conferences. He is author/coauthor of five books and 20 book chapters in the areas of communications. Prof. Vasilakos has served as General Chair, TPC Chair, TPC member (i.e INFOCOM, SECON, MOBIHOC) for many international conferences. He served or is serving as an Editor or/and Guest Editor for many technical journals including IEEE ToN, IEEE IEE TNSM, IEEE TSMC-B, IEEE TC, ACM TAAS, IEEE JSAC special issues of May 2009, Jan 2011, March 2011, IEEE Communications Magazine, ACM/ Springer WINET, MONET. He is founding Editor-in-Chief of the International Journal of Adaptive and Autonomous Communications Systems and the International Journal of Arts and Technology. He is General Chair of the Council of Computing of the European Alliances for Innovation. 